\title{
ON VARIETIES OF METABELIAN p-GROUPS, AND THEIR LAWS
}

\author{
WARREN BRISLEY \\ (Received 25 October 1965, revised 23 May 1966)
}

\section{Introduction}

The purpose of this article is to present some results on varieties of metabelian $p$-groups, nilpotent of class $c$, with the prime $p$ greater than $c$. After some preliminary lemmas in $\S 3$, it is established in $\S 4$, Theorem 3, that there is a simple basis for the laws of such a variety, and this basis is explicitly stated. This allows the description of the lattice of such varieties, and in $\S 5$, Theorem 4 , it is shown that each such variety has a two-generator member which generates it. Theorem 7 extends this result to show that there is in each such variety a two-generator critical group which generates it; this is established by the help of Theorem 5, which states that each critical group is a two-generator group, and Theorem 6 , which gives explicitly the varieties generated by the proper subgroups, by the proper quotient groups, and by the proper factor groups of such a critical group.

Some of the theorems and lemmas, with minor re-wording of their theses and proofs, also hold for the variety $\mathfrak{M}_{c}$ of all metabelian groups of class $c$; in particular, Theorem 1 can be modified to afford an independent proof of a result due to Magnus, quoted in [2], (and also [9], corollary 36.23 ), that the basic $c$-weight commutators of a reduced free group of $\mathfrak{M}_{\text {c }}$ are independent.

Some of the theorems do not generalise: for example, any generalisation of Theorem $\mathbf{5}$ is limited by the existence of 3-generator critical metabelian 3-groups.

\section{Notation and definitions}

As usual, the commutator $x^{-1} y^{-1} x y$ is written $(x, y)$, with left-normed commutators of weight $k$ being defined recursively by $\left(x_{1}, x_{2}, \cdots, x_{k}\right)$ $=\left(\left(x_{1}, x_{2}, \cdots, x_{k-1}\right), x_{k}\right), k>2$. For repeated symbols in a commutator, the notation $(x, n y)$ is used ( $n$ being a positive integer) where $(x, 1 y)=(x, y)$, and recursively $(x, n y)=((x,(n-1) y), y), n>1$; the obvious meaning is attached to the symbol $\left(x_{1}, n_{2} x_{2}, n_{3} x_{3}, \cdots, n_{8} x_{8}\right)$ where all the $n_{i}$ are non- 
negative integers, and at least one $n_{i}$ is not zero; the symbol could clearly be defined recursively. By $\gamma_{k}(G)$ we mean the $k^{\text {th }}$ term of the lower central series of $G$, i.e. the verbal subgroup $\gamma_{k}(G)=G p\left\{\left(x_{1}, x_{2}, \cdots, x_{k}\right) \mid x_{i} \in G\right.$, $i=1, \cdots, k\}$. The notation $G^{p}$ means the verbal subgroup $G p\left\{x^{p} \mid x \in G\right\}$, and the Frattini subgroup is denoted by $\Phi(G)$, where we know, if $G$ is a finite $p$-group, that $\Phi(G)=G p\left\{G^{p}, \gamma_{2}(G)\right\}$.

The varieties considered are subvarieties of $\mathfrak{M}_{c}$, the variety of all metabelian groups of class $c$. The phrase " $\mathfrak{B}$ is a variety of type $\mathfrak{M}(p, c)$ " is defined to mean that for the fixed prime $p>c, \mathfrak{B}$ has $p$-power exponent, is metabelian, and is of class $c$; also, that at least one group in $V$ is not of class $c-1$, so that there is, amongst the laws of $V$, a law of the form $\left(x_{1}, x_{2}, \cdots, x_{c}\right)^{p^{\alpha_{c}}}=1$, where $\alpha_{c}>0$. It is to be understood that $\alpha_{c}$ is minimal for this law in $\mathfrak{B}$, and the symbol $\alpha_{c}$ is used in this sense throughout.

The variety generated by a group $G$ is denoted by $\operatorname{Var}(G)$; the variety generated by its proper subgroups is denoted by $\operatorname{Var}(S-1) G$, and by all its proper factors by $\operatorname{Var}(Q S-1) G$. A finite group $G$ is called critical if $G \notin \operatorname{Var}(Q S-1) G$. The properties of varieties, and the correspondences amongst varieties, free groups, verbal subgroups, reduced free groups, and sets of laws of varieties, may all be found in [8] and [9].

Calculations will be carried out, almost entirely, in metabelian groups, i.e. groups for which $\gamma_{2}(G)$ is Abelian. In this case, we have that $\left(x_{1}, x_{2}, x_{3}, x_{4}\right)=\left(x_{1}, x_{2}, x_{4}, x_{3}\right)$, and the Jacobi-Witt identity becomes

The general rules

$$
(x, y, z)(y, z, x)(z, x, y)=1 \text {. }
$$

$$
\begin{aligned}
& (x y, z)=(x, z)(x, z, y)(y, z) \\
& (x, y z)=(x, z)(x, y)(x, y, z)
\end{aligned}
$$

are then sufficient to perform any of the calculations used. In particular, any product of commutators may be rearranged to an equivalent product of basic commutators, according to some ordering of the generators involved.

\section{Preliminary lemmas}

The first result is a lemma about possible manipulations in a commutative group; a corollary about certain laws in a variety of $p$-groups follows from it.

LEMma 1. Let $G$ be a group with commuting elements $W_{k}, k=1,2, \cdots n$ and containing no elements of prime order $\leqq n$. If the set $\left\{S_{\lambda}\right\}$ of relations hold:

$$
S_{\lambda}: \prod_{k=1}^{n} W_{k}^{\lambda^{k}}=1, \quad \lambda=1,2, \cdots, n
$$

then $W_{k}=1$ for each $k, k=1,2, \cdots n$. 
Proof. By raising the left-hand sides of the relations $S_{\lambda}$ to powers, taking inverses, and multiplying together, we can derive new relations of the form $R$ :

$$
R: \prod_{k=1}^{n} W_{k}^{\xi k}=1 .
$$

Now corresponding to the $\lambda^{\text {th }}$ relation of the set $\left\{S_{\lambda}\right\}$, we have the exponents of the $W_{k}$ as the $\lambda^{\text {th }}$ row of the matrix

$$
\left(\begin{array}{cccc}
1 & 1 & \cdots & 1 \\
2 & 2^{2} & \cdots & 2^{n} \\
3 & 3^{2} & \cdots & 3^{n} \\
\vdots & \vdots & & \vdots \\
n & n^{2} & \cdots & n^{n}
\end{array}\right)
$$

Clearly the set of $\xi_{k}$ in a relation of the form $R$ will correspond to the result, in some row, after some sequence of row operations has been carried out on this matrix, entries being considered over the ring of integers, (i.e. disallowing division). But from an explicit formula [1] for the Stirling numbers of the second kind, $S_{j}^{(i)}$, namely

$$
S_{i j}=i ! S_{j}^{(i)}=\sum_{t=0}^{i}(-1)^{i-t}\left(\begin{array}{l}
i \\
t
\end{array}\right) t^{j}
$$

(which yields $S_{i}^{(i)}=1, S_{j}^{(i)}=0$ for $i>j$ ), it is clear that ring operations alone suffice to bring the matrix, by row operations, to the form

$$
\left(\begin{array}{lllrll}
1 & 1 & 1 & 1 & \cdots & 1 \\
0 & 2 ! & 6 & 14 & \cdots & \\
0 & 0 & 3 ! & 36 & \cdots & \\
\vdots & \vdots & \vdots & \vdots & & \\
0 & 0 & 0 & 0 & \cdots & n !
\end{array}\right) .
$$

The entry in the $i^{\text {th }}$ row and $j^{\text {th }}$ column is $S_{i j}$, and the entries in a suitable row now correspond to the $\xi_{k}$ of some relation of the form $R$. In particular, since $W_{n}^{n !}=1$ is a law, and $G$ contains no elements of prime order $\leqq n$, it follows that $W_{n}=1$. Since the $r^{\text {th }}$ row of the matrix corresponds to the relation

$$
W_{r}^{r !} \prod_{k=r+1}^{n} W_{k}^{s_{r}}=1, \quad r \leqq n,
$$

an induction over $(n-r)$ completes the proof of the lemma.

We now turn to an immediate corollary, which deals with certain laws in a variety $\mathfrak{B}$ of $p$-groups. When dealing with laws in a variety, 
certain transformations applied to the laws (e.g. thorough replacement of one variable by another, thorough replacement of a variable by 1 , etc.) yield valid laws; essentially, such transformations are endomorphisms on the reduced free group of suitable rank. Such a transformation is termed "valid" in the following:

COROLIARY 1.1. Let $\mathfrak{B}$ be a variety of $p$-groups, with the lawe $\Pi_{k=1}^{n} W_{k}=1$, $n<p$, where the $W_{k}$ are distinct commuting words. If there is a set of valid transformations $\left\{\phi_{\lambda}\right\}, \lambda=1,2, \cdots, n$, such that $\phi_{\lambda}\left(W_{k}\right)=W_{k}^{\lambda^{k}}$, then $W_{k}=1$ is a law in $\mathfrak{B}$ for each $k: k=1,2, \cdots, n$.

Proof. By the transformations $\phi_{\lambda}$, we deduce the set $\left\{L_{\lambda}\right\}$ of laws of $\mathfrak{B}$ :

$$
L_{\lambda}: \prod_{k=1}^{n} W_{k}^{\lambda^{k}}=1, \quad \lambda=1,2, \cdots, n .
$$

Interpreting these laws as relations on the generators of a reduced free group of suitable rank, Lemma 1 applies, and the corollary follows. [An example of such a set of words and transformations is as follows: Let $\mathfrak{B}$ be a subvariety of $\Re_{c}$ (the variety of all groups nilpotent of class $c$ ), with $F$ a reduced free group on generators $x, y, z, w, \cdots$, and let $W_{k}=(x,(c-k-1) y, k z)^{\beta_{k}}$, $k=1,2, \cdots, c-1$. Then a set of endomorphisms with the required property is given by $\phi_{\lambda}$ being the substitution of $z^{\lambda}$ for $z$, leaving $x, y, z, \cdots$, fixed, in every word of $F$.]

We now turn to varieties of type $\mathfrak{M}(p, c)$, and first consider laws which involve two variables only, in commutator words of weight $c$. From corollary 1.1 we derive the further corollary:

COROLlary 1.2. Let $\mathfrak{B}$ be a variety of type $\mathfrak{M}(p, c)$. If $\mathfrak{B}$ has a law of the form

$$
\prod_{i=0}^{c-2}(x,(c-1-i) y, i x)^{\beta_{i}}=1,\left(\text { with some } \beta_{i} \neq 0\right)
$$

then $\mathfrak{B}$ also has the set of laws $\left\{L_{i}\right\}$,

$$
L_{i}:(x,(c-1-i) y, i x)^{p^{\mu}}=1, \quad i=0,1, \cdots c-2,
$$

where $\mu>0$, and $p^{\mu}$ is the g.c.d. of the non-zero $\beta_{i}$.

Proof. We may regard $(x,(c-1-i) y, i x)^{\beta_{i}}$ as a $W_{k}$ of corollary 1.I, with $k=i+1, n=c-1$, and the $\phi_{\lambda}$ being afforded by the substitution of $x^{\lambda}$ for $x$. It then follows that $(x,(c-1-i) y, i x)^{\beta_{i}}=1$ is a law for each $i$. Since $\mathfrak{B}$ is of type $\mathfrak{M}(p, c)$, clearly we may take $\beta_{i}=p^{\xi_{i}}$ for some $\xi_{i} \geqq 0$, (or else $\beta_{i}=0$, and the corresponding term is trivial in the original product). Further, if $(x,(c-1-i) y, i x)=1$ were a law, then a result due to Gupta and Newman [4] would require that, for any $G \in \mathfrak{B}$, the exponent of $\gamma_{\bullet}(G)$ 
divide the number $c(c-i)(c-2-i) !(c-1-i) ! i ! \prod_{k=1}^{i} k ! \prod_{t=1}^{c-2-i} t$ !. Since $p>c$, this is clearly not so, and thus $\xi_{i}>0$. Then for any non-zero $\beta_{i}$ of the original law, we have the law $L_{i}$ :

$$
L_{i}:(x,(c-1-i) y, i x)^{\beta_{i}}=1, \beta_{i}=p^{\xi_{i}}, \xi_{i}>0 .
$$

In $L_{i}$, substitute $x y$ for $x$ : then

$$
L_{i}^{\prime}: \prod_{s=0}^{i}(x,(c-1-i+s) y ;(i-s) x)^{\left(\begin{array}{l}
i \\
i
\end{array}\right) \beta_{i}}=1 .
$$

In $L_{i}$, substitute $x y$ for $y$ : then

$$
L_{i}^{\prime \prime}: \prod_{s=0}^{c-2-i}(x,(c-1-i-s) y,(i+s) x)^{\left({ }^{c-2-i}\right) \beta_{i}}=1 .
$$

By what we have already proved above, each of these laws may be appropriately "factored"; since $p$ divides neither $\left(\begin{array}{l}i \\ s\end{array}\right)$ nor $\left(\begin{array}{c}c-2-i \\ s\end{array}\right)$, we have for each non-zero $\beta_{i}$ the set of laws $\left\{S_{m}\right\}$

$$
\left\{S_{m}\right\}:(x,(c-1-m) y, m x)^{\beta_{i}}=1, \quad m=0,1,2, \cdots, c-2 .
$$

Since each non-zero $\beta_{i}$ is of the form $p^{\xi_{i}}, \xi_{i}>0$, we may take the minimal $\xi_{i}$, and the corollary has been proved.

It is worth noting that the following corollary, which will be used in $\S 4$, can be derived immediately from Gupta and Newman's result;

COROLlaRY 1.3. If $\mathfrak{B}$ is a variety of metabelian p-groups, of class $c$, with $p>c$, and the $n^{\text {th }}$-Engel law $(x, n y)=1$ holds in $\mathfrak{B}$, with $n<c$, then $\mathfrak{B}$ is of class $n+1$.

We now turn to laws involving more than two variables.

LEMMA 2. In a variety $\mathfrak{B}$ of type $\mathfrak{M}(p, c)$, there is no law of the form $\left(x_{1}, x_{2}, s_{3} x_{3}, \cdots, s_{t} x_{t}\right)^{\mu \mu}=1$, with $\mu<\alpha_{c}$, where the commutator is of weight $c$ in the $t$ distinct variables $x_{1}, x_{2}, \cdots, x_{t}, t \geqq 3$, and all $s_{i}>0$.

This lemma can be extended to the case of two variables by a corollary:

CoRollary 2.1. In a variety $\mathfrak{B}$ of type $\mathfrak{M}(p, c)$, there is no lawe of the form $(x,(c-1) y)^{p^{\mu}}=1$, with $\mu<\alpha_{c}$.

The proofs follow.

Proof of lemma 2. Since the result for $t=c$ is immediate by definition of $\mathfrak{B}$, we proceed by induction on $c-t$, the inductive hypothesis being that the lemma is true for $t=c, c-1, \cdots, c-r$, with $r \leqq c-4$. Then if there were a law of the above form for $t=c-r-1$, it could be written in the form $L$ :

$$
L:\left(x_{1}, x_{2}, \cdots, s_{c-r-2} x_{c-r-2}, i x_{c \rightarrow-1}\right)^{p^{\mu}}=1
$$

where the commutator is of weight $c$, and $i=s_{c \rightarrow-1}>0$. By substituting 
$x_{c \rightarrow-1} x_{c \rightarrow r}$ for $x_{c \rightarrow-1}$ wherever it occurs, and using $L$ to remove the term with $k=0$, we have the new law $L^{\prime}$;

$$
L^{\prime}: \prod_{k=1}^{i}\left(x_{1}, x_{2}, \cdots, x_{c \rightarrow-2},(i-k) x_{c \rightarrow-1}, k x_{c \rightarrow r}\right)^{\left(\begin{array}{l}
i \\
k
\end{array}\right) p^{\mu}}=1 .
$$

Lemma 1 now applies to $L^{\prime}, W_{k}$ being the power of the commutator with $k$ entries of $x_{c \rightarrow r}, n$ being $i$, and $\phi_{\lambda}$ being the substitution of $x_{c-r}^{\lambda}$ for $x_{c-r}$ throughout. Thus, in particular,

$$
\left(x_{1}, x_{2}, \cdots, x_{c \rightarrow-2},(i-1) x_{c-r-1}, x_{c-r}\right)^{i p^{\mu}}=1 \text {, }
$$

and since $p>c>i$, we have a contradiction to the inductive hypothesis if $\mu<\alpha_{c}$, and the lemma is established.

We turn to the corollary;

Proof of Corollary 2.1. In the proposed law $(x,(c-1) y)^{p^{\mu}}=1$, substitute $y z$ for $y$, giving the law

where

$$
(x,(c-1) y)^{p^{\mu}} \prod_{k=1}^{c-2} W_{k}(x,(c-1) z)^{p^{\mu}}=1
$$

$$
W_{k}=(x, y,(c-2-k) y, k z)^{\left(\begin{array}{c}
c-2 \\
k
\end{array}\right) n \mu}(x, z,(c-1-k) y,(k-1) z)^{\left(\begin{array}{c}
c-2 \\
k-1
\end{array}\right) p^{\mu}} .
$$

But the proposed law gives $\prod_{k=1}^{c-2} W_{k}=1$, to which Lemma 1 may be applied, $\phi_{\lambda}$ being the substitution of $z^{\lambda}$ for $z$; in particular,

$$
W_{c-2}=(x, y,(c-2) z)^{p^{\mu}}(x, z, y,(c-3) z)^{(c-2) p^{\mu}}=1 \text {. }
$$

In this law, interchange $x$ and $y$ and take inverses: this gives

$$
W_{c-2}^{\prime}=(x, y,(c-2) z)^{p^{\mu}}(z, y, x,(c-3) z)^{(c-2) p^{\mu}}=1 \text {. }
$$

Since $\mathfrak{B}$ is metabelian, we have the Jacobi identity

$$
(x, z, y)(z, y, x)(y, x, z)=1 \text {, }
$$

from which, since $\mathfrak{B}$ is of class $c$,

$$
(x, z, y,(c-3) z)(z, y, x,(c-3) z)(y, x,(c-2) z)=1 .
$$

Taking this relation with the law $W_{c-2} W_{c-2}^{\prime}=1$, we derive $(x, y,(c-2) z)^{c p^{\mu}}=1$, and since $p>c$, we have $(x, y,(c-2) z)^{p^{\mu}}=1$, so that $\mu \nless \alpha_{0}$ by Lemma 2 , and the corollary is proved.

From corollary 2.1, we have immediately:

CoRollary 2.2. In a variety $\mathfrak{B}$ of type $\mathfrak{M}(p, c)$, the laws $(x,(c-1) y)^{p^{\alpha}}=1$ and $\left(x_{1}, x_{2}, \cdots, x_{c}\right)^{p_{0}}=1$ are equivalent.

By taking Corollary 1.2 and Corollary 2.1 together, we have the stronger result: 
LEMMA 3. For a variety $\mathfrak{B}$ of type $\mathfrak{M}(p, c)$, any law of the form $\prod_{i=0}^{c-2}(x,(c-1-i) y, i x)^{\beta_{i}}=1$ can be assumed to have $\beta_{i}=0 \bmod p^{\alpha}$. for each $i$.

The next lemma will provide an inductive step to help establish a theorem about basic commutators in a reduced free group of a variety $\mathfrak{B}$ of type $\mathfrak{M}(p, c)$. Using the properties of metabelian groups, any commutator law can be shown to be equivalent to a law which involves basic commutators only, according to some ordering of the generators of the corresponding free group. Lemma 4 will be used later to establish Theorem 1, which deals with basic commutators; to state Lemma 4, we need the following notation.

Let $A(i, j)$ be a product of commutators of weight $c$, whose entries are from the set of $n$ variables $x_{1}, x_{2}, \cdots, x_{n}$, of the form

$$
A(i, j)=\prod_{q \in Q_{j}}\left(x_{i}, x_{j}, N_{j} x_{j}, N_{j+1} x_{j+1}, \cdots, N_{n} x_{n}\right)^{\alpha_{q}(i, j)}, \quad n \geqq i>j \geqq 1 .
$$

$Q_{j}$ is the set of all possible partitions of $c-2$ into $n-j+1$ parts $\left\{\beta_{j}, \beta_{j+1}, \cdots, \beta_{n}\right\}$ so that $\beta_{j}+\beta_{j+1}+\cdots+\beta_{n}=c-2, \beta_{k} \geqq 0$ for $k=j, j+1, \cdots, n ; q$ is the element of $Q_{j}$ with $\beta_{k}=N_{k}$, and $\alpha_{a}(i, j)$ is an integer.

Using this notation, we establish

LEMMA 4. If, for a variety $\mathfrak{B}$ of type $\mathfrak{M}(p, c)$, there is an integer $s, 2 \leqq s<n$ such that all laws of the form

$$
L(s): A(s+1, s) A(s+2, s) \cdots A(n, s)=1
$$

are consequences of the law $\left(x_{1}, x_{2}, \cdots, x_{c}\right)^{p^{\alpha}}=1$ in the sense that all $\alpha_{q}(k, s)$ in $L(s)$ are 0 mod $p^{\alpha}$, then the same is true for all laws of the form $L(s-1)$.

Proof. Any particular law of the form $L(s-1)$ may be explicitly written out as

$$
L(s-1): P(1) \prod_{q \in Q_{0-1}}\left(x_{s}, x_{s-1}, N_{s-1} x_{s-1}, \cdots, N_{n} x_{n}\right)^{\alpha_{q}(s, s-1)}=1
$$

where $P(1)$ is the case $\lambda=1$ for the product

$$
P(\lambda)=\prod_{k=0+1}^{n} \prod_{q \in Q_{s-1}}\left(x_{k}, x_{s-1}, N_{s-1} x_{s-1}, \cdots N_{n} x_{n}\right)^{\xi_{q}}
$$

with $\xi_{q}=\lambda^{N} \alpha_{q}(k, s-1), \lambda$ an integer, the elements $q$ of $Q_{s-1}$ being identified by the entries in the $(n-s+2)$-tuple $\left\{N_{s-1}, N_{0}, \cdots, N_{n}\right\}$. (The form $P(\lambda)$ is needed later.)

Substituting $x_{s}^{\lambda}$ for $x_{s}$ throughout $L(s-1)$, we derive the law $L^{\prime}$ :

$$
L^{\prime}: P(\lambda) \prod_{Q \in Q_{s-1}}\left(x_{s}, x_{s-1}, N_{s-1} x_{s-1}, \cdots, N_{n} x_{n}\right)^{\beta_{q}}=1
$$

where $\beta_{q}=\lambda^{N_{s}+1} \alpha_{q}(s, s-1)$. Then substituting $x_{s}$ for $x_{s-1}$ throughout $L^{\prime}$, we have $L^{\prime \prime}$ : 


$$
L^{\prime \prime}: \prod_{k=s+1}^{n} \prod_{q \in Q_{n-1}}\left(x_{k}, x_{s},\left(N_{s-1}+N_{s}\right) x_{s}, N_{s+1} x_{s+1}, \cdots, N_{n} x_{n}\right)^{\xi_{q}}=1 .
$$

Now this last law $L^{\prime \prime}$ may be written as a law of the form $L(s)$ by collecting terms, and considering the product over the set $Q_{s}$ of partitions. Thus, for each element of $Q$ of the form $\left\{\beta_{s}, N_{s+1}, N_{s+2}, \cdots, N_{n}\right\}$, and each $k>s$, we have in $L^{\prime \prime}$ a term of the form

$$
\left(x_{k}, x_{s}, \beta_{8} x_{s}, N_{8+1} x_{8+1}, \cdots, N_{n} x_{n}\right)^{\eta}
$$

where $\eta=\sum \xi_{q}, \xi_{q}=\lambda^{N_{a}} \alpha_{q}(k, s-1), q$ is the element of $Q_{s-1}$ of the form $\left\{N_{s-1}, N_{s}, \cdots, N_{n}\right\}$, and the sum ranges over $N_{s},\left(N_{s}=0,1, \cdots, \beta_{s}\right)$, with $q$ restricted by $N_{s-1}+N_{s}=\beta_{s}$. But by the inductive hypothesis, since $L^{\prime \prime}$ is now of the form $L(s)$ we have $\eta=0 \bmod p^{\alpha}$. Taking $\lambda=1,2, \cdots, \beta_{s}+1$, we have the set $E$ of equations:

$$
E:\left(\begin{array}{ccccc}
1 & 1 & 1 & \cdots & 1 \\
1 & 2 & 2^{2} & & 2^{\beta_{s}} \\
1 & 3 & 3^{2} & & 3^{\beta_{s}} \\
\vdots & \vdots & \vdots & & \vdots \\
1 & \left(\beta_{s}+1\right) & \left(\beta_{s}+1\right)^{2} & \cdots & \left(\beta_{s}+1\right)^{\beta_{s}}
\end{array}\right)\left(\begin{array}{c}
\alpha_{0} \\
\alpha_{1} \\
\vdots \\
\vdots \\
\alpha_{\beta_{s}}
\end{array}\right)=0 \bmod p^{\alpha_{a}}
$$

where $\alpha_{i}$ denotes $\alpha_{a}(k, s-1)$ for $q=\left\{\left(\beta_{s}-i\right), i, N_{s+1}, \cdots, N_{n}\right\} \in Q_{s-1}$. Since the determinant of the matrix is $\prod_{r=1}^{\beta_{a}} r$ !, and $p>c>\beta_{s}$, each of the $\alpha_{i}=0 \bmod p^{\alpha_{i}}$, and hence, by considering all the elements of $Q_{b}$, we have $\alpha_{q}(k, s-1)=0 \bmod p^{\alpha}$ for all $k>s$ and all $q \in Q_{s-1}$. Thus, $P(1) \equiv 1$, and the law $L(s-1)$ reduces to

$$
L: \prod_{q \in Q_{s-1}}\left(x_{s}, x_{s-1}, N_{s-1} x_{s-1}, \cdots, N_{n} x_{n}\right)^{\alpha_{q}(s, s-1)}=1 .
$$

By substituting $x_{s+1}^{\lambda}$ for $x_{s+1}$ throughout $L$, we have

$$
\prod_{q \in Q_{s-1}}\left(x_{s}, x_{s-1}, N_{s-1} x_{s-1}, \cdots, N_{n} x_{n}\right)^{\mu_{a}}=1
$$

with $\mu_{q}=\lambda^{N_{a+1}} \alpha_{a}(s, s-1)$. Substitution of $x_{s+1}$ for $x_{s}$ throughout this last law gives

$$
\prod_{q \in Q}\left(x_{s+1}, x_{s-1}, N_{s-1} x_{s-1},\left(N_{s}+N_{s+1}\right) x_{s+1}, \cdots, N_{n} x_{n}\right)^{\mu_{q}}=1,
$$

and substitution of $x_{s}$ for $x_{s-1}$ throughout this then gives

$$
\prod_{a \in Q_{s-1}}\left(x_{s+1}, x_{s}, N_{s-1} x_{s},\left(N_{s}+N_{s+1}\right) x_{s+1}, \cdots, N_{n} x_{n}\right)^{\mu_{a}}=1 \text {. }
$$

By collecting terms, and rearranging, this last law may be put in a form $L^{\prime}(s)$ :

$$
L^{\prime}(s): \prod_{Q \in Q_{s}}\left(x_{s+1}, x_{s}, N_{s} x_{s}, \beta_{s+1} x_{s+1}, \cdots, N_{n} x_{n}\right)^{\delta_{r}}=1
$$


where $r=\left\{\beta_{s}, \beta_{s+1}, \cdots, \beta_{n}\right\} \in Q_{s}$, and $r$ is related to the elements $q$ of $Q_{s-1}$ by $\beta_{s}=N_{s-1}, \quad \beta_{s+1}=N_{s}+N_{s+1}, \quad \beta_{s+2}=N_{s+2}, \cdots, \beta_{n}=N_{n}$. The indices $\delta_{r}$ are given by $\delta_{r}=\sum_{N_{s+1}} \lambda^{N_{s+1}} \alpha_{q}(s, s-1)$, and by hypothesis each $\delta_{r}=0 \bmod p^{\alpha}$.

Putting $\lambda=1,2, \cdots, \beta_{s+1}+1$, a set of equations of the same form as $E$ is obtained, and the same argument then applies, since $p>c>\beta_{8+1}$, and so each $\alpha_{q}(s, s-1)=0 \bmod p^{\alpha_{e}}$.

Thus, all indices in $L(s-1)$ have been shown to be zero $\bmod p^{\alpha}$, and the lemma is proved.

So far, all the lemmas have been concerned with commutators of weight $c$ in a variety $\mathfrak{B}$ of class $c$. If all the laws of $\mathfrak{B}$ are to be examined, we need a result which will allow some induction along the weight of commutators occurring in a law. The last lemma of this section provides that step:

Lemma 5. Let the variety $\mathfrak{B}$ of type $\mathfrak{M}(p, n+2)$ be generated by a reduced free group $F$. Then if $(x,(n+1) y)^{p^{x}}=1$ is a law in $\mathfrak{B}$, and $(x, n y)^{p^{r}}=1$ is a law in $F / \gamma_{n+2}(F)$, then $(x, n y)^{p^{r}}=1$ is a law in $\mathfrak{B}$.

(Note: from the proof, the lemma is true for $n=0$, if we interpret $(x, 0 y)$ as $x)$.

Proor. By Corollary 2.2, we have that the pair of laws

$$
\left(x_{1}, x_{2}, \cdots, x_{n+2}\right)^{p x}=1 \text { and }(x,(n+1) y)^{p x}=1
$$

are equivalent in $\mathfrak{B}$, and that the pair

$$
\left(x_{1}, x_{2}, \cdots, x_{n+1}\right)^{p^{r}}=1 \text { and }(x, n y)^{p^{r}}=1
$$

are equivalent in $\operatorname{Var}\left(F / \gamma_{n+2}(F)\right)$.

If $(x, n y)^{p^{r}}$ was not always trivial in $F$, there would have to be at least two free generators $x_{i}, x_{j}$ of $F$ such that $\left(x_{i}, n x_{j}\right)^{p^{r}} \neq 1$; but $\left(x_{i}, n x_{j}\right)^{p^{p}} \in \gamma_{n+2}(F)$ by hypothesis, so $\left(x_{i}, n x_{j}\right)^{p^{p}}$ would be a product of commutators of weight $n+2$ in the generators $x_{1}, x_{2}, x_{3}, \cdots$ of $F$, and this yjelds a law in $F$ and hence in $\mathfrak{B}$. By putting $x_{k}=1$ whenever $k$ is neither $i$ nor $j$, we could then deduce a two variable law in $x_{i}$ and $x_{j}$. Reading $x$ for $x_{i}$ and $y$ for $x_{j}$, we have that there would be a law in $\mathfrak{B}$ of the form $L$ :

$$
L:(x, n y)^{p^{\tau}}=\prod_{k=1}^{n+1}(x, k y,(n+1-k) x)^{\beta_{k}}
$$

By commuting once with $y$, we see that $r \geqq \alpha$, and hence $(x, n y)^{p^{*}}$ becomes $(x, n y)^{\lambda n_{p^{r}}}$ under the substitution of $y^{\lambda}$ for $y$. By first writing $L$ in the form $L^{\prime}$ :

$$
\begin{aligned}
L^{\prime}: \prod_{k=1}^{n+1} W_{k}=1, W_{k}= & (x, k y,(n+1-k) x)^{\beta^{k}}, \\
& k \neq n, W_{n}=(x, n y)^{-p^{*}}(x, n y, x)^{\beta_{n}}
\end{aligned}
$$


we deduce, using corollary 1.1 with $\phi_{\lambda}$ being substitution of $y^{\lambda}$ for $y$, that $W_{k}=1$, and hence by Lemma 3 that $\beta_{k}=0 \bmod p^{\alpha}$, for each $k \neq n$. The only non-trivial part of the law remaining is $W_{n}=1$, i.e.

$$
(x, n y)^{-p^{r}}(x, n y, x)^{\beta_{n}}=1 \text {. }
$$

By using the substitution of $x^{-1}$ for $x$ we deduce that $(x, n y)^{p^{r}}=1$ is a law in $\mathfrak{B}$, and of course $\beta_{n}=0 \bmod p^{\alpha}$.

\section{Theorems on laws}

In this section, we consider the laws which a variety $\mathfrak{B}$ of type $\mathfrak{M}(p, c)$ may have. It is found that these laws have a basis which may be stated in terms of the maximum exponents of the lower central series of groups in $\mathfrak{B}$.

THEOREM 1. Let $F_{n}$ be a reduced free group (of a variety $\mathfrak{B}$ of type $\mathfrak{M}(p, c)$ ) of finite rank $n$, on the free generators $g_{1}, g_{2}, \cdots, g_{n}$, with ordering $g_{1}<g_{2}<\cdots<g_{n}$. Then the basic commutators, of weight $c$, in these generators, are independent.

Proof. The proof depends on the fact that the free generators of $F_{n}$ are such that any relation amongst them is a law in $F_{n}(\mathfrak{B})([8],[2])$.

Take the forms $A(i, j)$ as defined for the purposes of Lemma 4 , with symbols $g_{1}, g_{2}, \cdots, g_{n}$; by the ordering $g_{1}{ }^{\prime}<g_{2}<\cdots<g_{n}$, any relation amongst the basic $c$-weight commutators may be written in the form $(R)$ :

$$
\text { (R) }: T_{1} T_{2} \cdots T_{n-1}=1 \text {, with } T_{s}=\prod_{\substack{k \\ n \geqq k>s}} A(k, s) \text {. }
$$

This relation gives a law in $F_{n}(\mathfrak{B})$, and hence in $\operatorname{Var}\left(F_{n}(\mathfrak{B})\right)$, which is a variety of the type under consideration. By substituting 1 for the symbols $g_{1}, g_{2}, \cdots, g_{n-2}$ in this law, we have $T_{n-1}=1$ is a law, and so $T_{1} T_{2} \cdots T_{n-2}=1$ is a law. Continuing by substituting 1 for $g_{1}, g_{2}, \cdots, g_{n-3}$, and so on, we have that each of $T_{s}=1$ is a law, for $s=n-1, n-2, \cdots 1$. But $T_{n-1}=A(n, n-1)$, so by Lemma 3 (using symbols $x$ for $g_{n}$ and $y$ for $\left.g_{n-1}\right)$, the law $T_{n-1}=1$ is a direct consequence of the law $\left(x_{1}, x_{2}, \cdots, x_{c}\right)^{p^{\alpha_{c}}}=1$, in the sense that each exponent of each commutator is $0 \bmod p^{\alpha}$. Lemma 4 now provides the inductive step, and hence all the exponents of all the distinct commutators in each factor $T$, of the relation $(R)$ are $0 \bmod p^{\alpha_{e}}$, the left side of $R$ is identically 1 , and the theorem is established.

Considering the remarks preceding Lemma 4, we can restate Theorem 1 as a theorem about the laws of a variety $\mathfrak{B}$ of type $\mathfrak{M}(p, c)$, since $\mathfrak{B}$ is in fact generated by $F_{c}(\mathfrak{B})([5],[2])$. Thus:

THEOREM 2. For a variety $\mathfrak{B}$ of type $\mathfrak{M}(p, c)$, all laws of the form 
$\Pi_{i} W_{i}^{\beta_{i}}=1$, where $W_{i}$ are commutators of weight $c$, in $n$ variables, are consequences of the single law in $c$ variables:

$$
\left(x_{1}, x_{2}, \cdots, x_{c}\right)^{\alpha_{c}}=1 .
$$

We can now give the form for a basis of the laws of any given variety of the type $\mathfrak{M}(p, c)$, and name any such variety by giving its relevant exponents. This is done in Theorem 3. The existence of, and generation of, such varieties, is dealt with in $\S \mathbf{5}$.

THEOREM 3. The laws of a variety $\mathfrak{B}$ of type $\mathfrak{M}(p, c)$ can all be derived from the following basis:

(i) the metabelian law $\left(\left(x_{1}, x_{2}\right),\left(x_{3}, x_{4}\right)\right)=1$

(ii) the nilpotency law $\left(x_{1}, x_{2}, \cdots, x_{c}, x_{c+1}\right)=1$

(iii) a set of two-variable laws, $c$ in number, of the form

$$
x^{p^{\alpha_{1}}}=1, \quad(x, k y)^{p^{\alpha_{k+1}}}, \quad k=1,2, \cdots, c-1
$$

in which $\alpha_{1} \geqq \alpha_{2} \geqq \cdots \geqq \alpha_{0} \geqq 1$.

Proof. It is known ([5], [2]) that there must be at least one reduced free group $F$ in $\mathfrak{B}$ such that $\mathfrak{B}=\operatorname{Var}(F)$. Now $\mathfrak{B}$ certainly has laws of the form stated in the theorem, as we know by corollary 1.3, and we will take the $\alpha_{i}$ as minimal. Then $F / \gamma_{0}(F)$ has the law $(x,(c-2) y)^{p^{\alpha_{0-1}}=1 \text {, since }}$ $F / \gamma_{c}(F) \in \mathfrak{B}$; by Lemma $5, \alpha_{c-1}$ is minimal for $F / \gamma_{c}(F)$, and by corollary 2.2, this is equivalent to the law $\left(x_{1}, x_{2}, \cdots, x_{c-1}\right)^{p^{\alpha} x_{0-1}}=1$ in $\operatorname{Var}\left(F / \gamma_{c}(F)\right)$; by Theorem 2, any law of $\operatorname{Var}\left(F / \gamma_{c}(F)\right)$ of the form $\Pi_{k} W_{k}^{\beta_{k}}=1$, where the $W_{k}$ are commutators of weight $c-1$, can be derived from it. Lemma 5 now provides the inductive steps necessary to establish:

"For all $k<c$,

$$
(x,(c-k) y)^{p^{\alpha}-k+1}=1
$$

is a basis law in $\operatorname{Var}\left(F / \gamma_{c-k+2}(F)\right)$ in the sense that all laws of the form $\Pi W_{i}^{\beta_{i}}=1, W_{i}$ commutators of weight $c-k+1$, follow from it."

Consider now $F$, and $\mathfrak{B}$, and take any $n$-variable law of $\mathfrak{B}$. Then this law may be written in the form $L$ :

$$
L: x_{1}^{r_{1}} x_{2}^{r_{2}} \cdots x_{n}^{r_{n}} P_{2} P_{3} \cdots P_{c}=1
$$

where each $P_{i}$ is a product of commutators of weight $i$. By substituting $x_{i}=1$, for all $i \neq k$, we have $r_{k}=0 \bmod p^{\alpha_{1}}$ for each $k$, and the law remaining is $P_{2} P_{3} \cdots P_{c}=1$. But this law holds also for $\operatorname{Var}\left(F / \gamma_{3}(F)\right) \subseteq \mathfrak{B}$, and hence $P_{2}=1$ is a law which follows from $(x, y)^{p^{\alpha}}=1$ and the metabelian law in the strict sense of Theorem 1 and Theorem 2, (i.e. if $P_{2}$ is written in the form $\Pi_{k} W_{k}^{\beta_{k}}$, all $W_{k}$ basic commutators of weight 2 , then $\beta_{k}=0$ $\left.\bmod p^{\alpha_{2}}\right)$. Thus $P_{2}=1$ is a law in $\mathfrak{B}$. The obvious induction, using Theorem 2 
at each stage, then gives that the whole law $L$ follows from the basis explicitly stated in the theorem, and the theorem follows.

From Theorem 3 , we may name any given variety $\mathfrak{B}$ of type $\mathfrak{M}(p, c)$ by the minimal indices in the basis laws, writing $\mathfrak{B}=\left[\alpha_{1}, \alpha_{2}, \cdots, \alpha_{c}\right]$. Clearly the varieties of type $\mathfrak{M}(p, c)$ for $p, c$ fixed, form a lattice under the obvious rules. For fixed $p$, and $k<c$, we may include all varieties of type $\mathfrak{M}(p, k)$ in the lattice; by interpreting the symbols $\left[\alpha_{1}, \alpha_{2}, \cdots, \alpha_{k}\right]$ and $\left[\beta_{1}, \beta_{2}, \cdots, \beta_{c}\right]$ as equivalent when $\alpha_{i}=\beta_{i}$ for $i=1,2, \cdots, k$ and $\beta_{i}=0$ for $i=k+1, k+2, \cdots, c$, (which is consistent in the light of corollary 1.3), the obvious rules then become

$$
\left[\beta_{1}, \beta_{2}, \cdots, \beta_{\mathrm{o}}\right] \vee\left[\beta_{1}^{\prime}, \beta_{2}^{\prime}, \cdots, \beta_{c}^{\prime}\right]=\left[\max \left(\beta_{1}, \beta_{1}^{\prime}\right), \cdots, \max \left(\beta_{c}, \beta_{\mathrm{c}}^{\prime}\right)\right]
$$
and

$$
\left[\beta_{1}, \beta_{2}, \cdots, \beta_{c}\right] \wedge\left[\beta_{1}^{\prime}, \beta_{2}^{\prime}, \cdots, \beta_{c}^{\prime}\right]=\left[\min \left(\beta_{1}, \beta_{1}^{\prime}\right), \cdots, \min \left(\beta_{c}, \beta_{c}^{\prime}\right)\right] .
$$

\section{Theorems on generation and critical groups}

Theorem 4 establishes the existence of each variety, and its generation; Theorems 5 and 6 deal with critical groups, and Theorem 7 deals with the generation of each variety by a critical group in it.

THEOREM 4. Any given variety $\left[\alpha_{1}, \alpha_{2}, \cdots, \alpha_{c}\right]$ of type $\mathfrak{M}(p, c)$ can be generated by a two-generator group, and hence by its free group of rank 2.

PROOF. The following group $G$, of order $p^{\alpha_{1}+2 \alpha_{3}+\cdots+\alpha_{e}}$ may be constructed, as shown in the Appendix:

$$
\begin{aligned}
& G=G p\left\{a, b \mid a^{p_{1}}=b^{\alpha_{2}}=\right.(a, b)^{p^{\alpha_{2}}}=\cdots=(a,(c-1) b)^{p \alpha_{o}}=(a, c b)=1, \\
&(a, b, a)=1, \text { metabelian }\} .
\end{aligned}
$$

A simple calculation shows that $G \in\left[\alpha_{1}, \alpha_{2}, \cdots, \alpha_{c}\right]$ and that these indices are minimal for $G$ and hence for $\operatorname{Var}(G)$. Since Theorem 3 applies to $\operatorname{Var}(G)$, we have $\operatorname{Var}(G)=\left[\alpha_{1}, \alpha_{2}, \cdots, \alpha_{c}\right]$.

We now examine critical groups, and can immediately state:

THEOREM 5. When $p>c>1$, any metabelian critical p-group of class $c$ is a two-generator group.

Proof. Let $H$ be such a critical group. Then Theorem 4 applies to $\operatorname{Var}(H)$, so that there is a two-generator group $G$, with $\operatorname{Var}(H)=\operatorname{Var}(G)$. Then by a theorem of B. H. and H. Neumann (see [7]), $H$ can be generated by $n$ generators, $n \leqq 2$.

We pass immediately to

THEOREM 6. Let $H$ be a metabelian p-group of class $c, p>c>1$, with $\operatorname{Var}(H)=\left[\alpha_{1}, \alpha_{2}, \cdots, \alpha_{c}\right]$. If $H$ is a two-generator group, then 
(i) $\operatorname{Var}(S-1) H=\left[\alpha_{1}, \beta_{2}, \cdots, \beta_{c-1}, \alpha_{c}-1\right]$ where $\beta_{k}=\max \left(\alpha_{k}-1, \alpha_{k+1}\right)$. If $H$ is critical, with monolith $N$, then

(ii) $\operatorname{Var}(H / N)=\left[\beta_{1}, \beta_{2}, \cdots, \beta_{c-1}, \alpha_{c}-1\right]$

(iii) $\operatorname{Var}(Q S-1) H=\operatorname{Var}(S-1) H \supseteqq \operatorname{Var}(H / N)$.

Proof. We prove (i) first. Since $H$ has two generators, we can designate any maximal subgroup $S$ of $H$ as $S=G p\{g, \Phi(H)\}$, where $\Phi(H)$ is the Frattini subgroup of $H$, and $g$ is some element of $H$. It is clear that if $(x,(i-1) y) \in \gamma_{i}^{p} \gamma_{i+1}(i>1)$ then $(x, i y) \in \gamma_{i+1}^{p} \gamma_{i+2}$. A simple calculation, using the forms $x=g^{8} f_{1}, y=g^{t} f_{2} ; f_{1}, f_{2} \in \Phi(H)$, shows that $(x, y) \in \gamma_{2}^{p} \gamma_{3}$, so we have by induction that $(x, i y) \in \gamma_{i+1}^{p} \gamma_{i+2}(i \geqq 1)$. Thus, for all proper subgroups $S$ of $H$, we have laws giving $\operatorname{Var}(S) \subseteq\left[\alpha_{1}, \beta_{2}, \cdots, \beta_{c-1}, \alpha_{c}-1\right]$. That there are proper subgroups of $H$ such that each of these indices is minimal is established as follows:

Consider first the case where $\alpha_{i}=\alpha_{i+1}$ for a particular $i>1$. Then in $H$ there must be two elements $g_{1}$ and $g_{2}$ such that $\left(g_{1}, i g_{2}\right)$ has order $p^{\alpha_{i+1}}$. But $S_{1}=G p\left\{\left(g_{1}, g_{2}\right), g_{2}\right\} \subset H$, since $\left(g_{1}, g_{2}\right) \in \Phi(H)$, and $H$ is twogenerator. Taking $\left(g_{1}, g_{2}\right)$ for $x$, and $g_{2}$ for $y$, we have a contradiction to $(x,(i-1) y)^{p^{\alpha_{i}-1}}$ being trivial in $S_{1}$. Consider next the case $\alpha_{i}>\alpha_{i+1}$ for some $i>2$; there must be elements $g_{3}$ and $g_{4}$ in $H$ such that $\left(g_{3},(i-1) g_{4}\right)$ has order $p^{\alpha_{i}}$. Then $S_{2}=G p\left\{g_{3}^{p}, g_{4}\right\} \subset H$, and using $g_{3}^{p}$ for $x, g_{4}$ for $y$, we have $(x,(i-1) y)^{p^{\alpha_{i}-2}}=\left(g_{3},(i-1) g_{4}\right)^{p_{i}-1}$ not trivial in $S_{2}$. Finally, there must be an element of order $p^{\alpha_{1}}$ in $H$, which by itself generates a proper subgroup of $H$. Thus the result (i) follows.

We next consider $H$ critical, in which case it is a two-generator group, by Theorem 5 , so that (i) still applies to $H$.

(ii): We note first that for all $x$ and $y$ in $H,(x,(c-1) y)^{p^{\alpha} e^{-1}}$ is central, of order $p$ if not trivial; since $N$ is the monolith of $H$, it follows that $N$ is the corresponding verbal subgroup, and so $(x,(c-1) y)^{p^{\alpha} a^{-1}}=1$ is a law in $H / N$. Since it is not a law in $H$, there exist elements $g_{5}, g_{6}$ in $H$ such that $\left(g_{5},(c-1) g_{6}\right)^{p^{\alpha_{c}-2}}$ is of order $p^{2}$, hence not in $N$, and so $(x,(c-1) y)^{p^{\alpha} a^{-2}}$ is not always trivial in $H / N$.

Consider now the case when $\alpha_{c-1}>\alpha_{c}$. Then

$$
\left((x,(c-2) y)^{p\left(\alpha_{c-1}-1\right)}, z\right)=(x,(c-2) y, z)^{p^{\left(\alpha_{c-1}-1\right)}}=1
$$

in $H$. [The equivalence of the two- and three-variable laws is dealt with by corollary 2.2]. Thus, $(x,(c-2) y)^{p\left(\alpha_{e-1}-1\right)}$ is always central, and of order $p$ when not trivial, and hence lies in $N$, so that $(x,(c-2) y)^{p\left(\alpha_{0-1}-1\right)}=1$ is a law in $H / N$. Since there are elements $g_{7}, g_{8}$ in $H$ such that $\left(g_{7},(c-2) g_{8}\right)^{p^{\left(\alpha_{0-1}-2\right)}}$ is of order $p^{2}$, and hence outside $N$, the index $\left(\alpha_{c-1}-1\right)$ is minimal for $\operatorname{Var}(H / N)$ when $\alpha_{c-1}>\alpha_{c}$.

The case when $\alpha_{n-1}=\alpha_{c}$ is transparent: there are elements $g_{9}, g_{10}$ 
in $H$ such that $\left(g_{9},(c-1) g_{10}\right)^{p^{\alpha}-1} \neq 1$, i.e. $\left(g_{9},(x-2) g_{10}\right)^{p^{\alpha}-1}$ is not central, and hence lies outside $N$, so that $(x,(c-2) y)^{p^{\left(x_{o-1}-1\right)}}$ is not always trivial modulo $N$.

Thus, $\beta_{c-1}$ is the minimal index for $\operatorname{Var}(H / N)$; the rest of the $\beta_{k}$ for $k>1$ follow by induction. Whenever $\alpha_{i}=\alpha_{i+1}$, the centrality argument applies as above, but whenever $\alpha_{i}>\alpha_{i+1}$, then $(x,(i-1) y)^{p^{\alpha_{i}-1}}$ is central, of order $p$ or less, and so is trivial modulo $N$. In that case there are elements (as $g_{7}, g_{8}$ above) so that $(x,(i-1) y)^{p^{\alpha_{1}-2}}$ is not a law in $\operatorname{Var}(H / N)$.

Finally, if $\alpha_{1}>\alpha_{2}$, then $x^{p^{\alpha_{1}-1}}$ is always central, of order $p$ if not trivial, and hence lies in $N$. Thus $x^{p^{\alpha_{1}-1}}$ is always trivial modulo $N$. But since there must be an element $x^{p_{1}^{\alpha_{1}-2}}$ of order $p^{2}$, hence not in $N$, we have that $x^{\alpha^{\alpha_{1}-2}}$ is not always trivial modulo $N$, and $x^{p^{\alpha_{1}-1}}=1$ is appropriate for $H / N$.

If $\alpha_{1}=\alpha_{2}$, we have that if $x^{p^{\alpha_{1}-1}}$ is always trivial modulo $N$, then $x^{p^{\alpha_{2}-1}}$ is always central, and hence $\left(x^{p^{\alpha_{1}-1}}, y\right)=1$ is a law of $H$, and this is equivalent to the law $\prod_{i=0}^{c-2}(x, y, i x)^{\left(p^{\alpha_{1}-1}\right)}=1$. But (by Corollary 1.1), this would imply $(x, y)^{p^{\alpha_{1}-1}}=1$ as a law of $H$, which is a contradiction. We conclude that $x^{p^{\beta_{1}}}=1$ is the appropriate law for $H / N$, and so (ii) is established.

The result (iii) follows from (i) and (ii) since

$$
\operatorname{Var}(Q S-1) H=\operatorname{Var}(S-1) H \vee \operatorname{Var}(H / N),
$$

$H$ being monolithic, and the theorem is proved.

It is worth noting that if $\alpha_{1}=\beta_{1}$, which occurs if and only if $\alpha_{1}=\alpha_{2}$, we are forced to $\operatorname{Var}(Q S-1) H=\operatorname{Var}(S-1) H=\operatorname{Var}(H / N)$. Two corollaries also follow from Theorem 6; examples of the properties they deal with are to be found in an earlier paper [3].

CoRollary 6.1. If $\operatorname{Var}(H)$ is of type $\mathfrak{M}(p, c)$, and $H$ is critical, then at most $c-2$ varieties $\mathfrak{B}_{i}$ may be found such that

$$
\operatorname{Var}(H) \supset \mathfrak{B}_{1} \supset \mathfrak{B}_{2} \supset \cdots \supset \mathfrak{B}_{k} \supset \operatorname{Var}(Q S-1) H,
$$

the inclusions being proper.

Corollary 6.2. If $\operatorname{Var}(H)$ is of type $\mathfrak{M}(p, c)$, then $\operatorname{Var}(Q S-1) H$ is maximal in $\operatorname{Var}(H)$ if and only if it is the unique maximal subvariety of $\operatorname{Var}(H)$.

The final theorem establishes the existence of critical groups.

THEOREM 7. For any given variety $\left[\alpha_{1}, \alpha_{2}, \cdots, \alpha_{c}\right]$ of the type $\mathfrak{M}(p, c)$, there exists at least one critical group $H$ such that $\operatorname{Var}(H)=\left[\alpha_{1}, \alpha_{2}, \cdots, \alpha_{c}\right]$.

Proof. (We note first that if $c=1$, the cyclic group is critical, and so consider $c>1$ ). For the given variety $\mathfrak{B}=\left[\alpha_{1}, \alpha_{2}, \cdots, \alpha_{c}\right]$, we determine 
those positions where $\alpha_{i}>\alpha_{i+1}$, and so determine the subscripts $(s 1),(s 2), \cdots(s n)$, that

$$
\begin{gathered}
\alpha_{1}=\alpha_{2}=\cdots=\alpha_{(s 1)}=e_{1} \\
\alpha_{s 1+1}=\alpha_{s 1+2}=\cdots=\alpha_{s 2}=e_{2} \\
\cdots \\
\alpha_{s k}=\alpha_{s k+1}=\cdots=\alpha_{s n}=e_{n},
\end{gathered}
$$

with $k=n-1,(s n)=c, e_{1}>e_{2}>\cdots>e_{n} \geqq 1$.

Let $G_{1}$ be the group which was constructed for the proof of Theorem 4: we use the notation $A_{1}=a, A_{i}=(a,(i-1) b)$, to write

$$
\begin{array}{r}
G_{1}=G p\left\{a, b \mid a^{p_{1}^{\alpha_{1}}}=b^{p^{\alpha_{2}}}=A_{2}^{p^{\alpha_{2}}}=\cdots=A_{o}^{p^{\alpha_{o}}}=1,\left(A_{i}, A_{j}\right)=1\right. \\
\text { for all } \left.i, j, A_{c+1}=1\right\} .
\end{array}
$$

Then $\operatorname{Var}\left(G_{1}\right)=\left[\alpha_{1}, \alpha_{2}, \cdots, \alpha_{c}\right]$. By a simple calculation it is immediate that $A_{(s 1)}^{p\left(\varepsilon_{1}-1\right)}, A_{(s 2)}^{p\left(e_{2}-1\right)}, \cdots, A_{(e n)}^{p\left(e_{n}-1\right)}$ are all central. Hence, by factoring out the product of the normal subgroups $N_{i}, N_{i}=G p\left\{A_{(s i)}^{p\left(e_{i}-1\right)} A_{(s i)}^{-p\left(\theta_{i}-1\right)}\right\}$, we have a group $G_{2} \simeq G p\{a, b \mid R\}$ where $R$ includes all the relations in $G_{1}$, and also the relations $A_{(s 1)}^{p^{\left(e_{1}-1\right)}}=\cdots=A_{(s n)}^{p\left(e_{n}-1\right)}$. It is easily verified that $\operatorname{Var}\left(G_{2}\right)$ is still $\mathfrak{B}$. (In the extreme case that $\alpha_{1}=\alpha_{2}=\cdots=\alpha_{c}, G_{2}=G_{1}$ ). Consider now the set of quotient groups $G_{2} / K$ of $G_{2}$, where $K$ is normal in $G_{2}$. Since this set includes both $G_{2}$ and $\{1\}$, there must be at least one group $H$ in the set which has the property $\operatorname{Var}(H)=\mathfrak{B}$, and is minimal with this property. (Thus if $N$ is any proper normal subgroup of $H, \operatorname{Var}(H / N)$ is a proper subvariety of $\mathfrak{B}$.)

We now assert that $H$ is critical.

Clearly $H$ is a two-generator group, inheriting all the relations of $G_{2}$, and we adopt the same notation $a, b, A_{i}$ for it. But part (i) of Theorem 6 was proved using only that $H$ was a two-generator group, hence

$$
\operatorname{Var}(S-1) H=\left[e_{1}, e_{1}, \cdots, e_{1}-1, e_{2}, \cdots, e_{2}-1, \cdots e_{n}, e_{n}-1\right]
$$

and so $H \notin \operatorname{Var}(S-1) H$. Suppose $H$ is not critical; then by a theorem of Weichsel [10] and Kovács and Newman [7], $H$ belongs to the variety generated by its proper quotient groups, and from this we derive a contradiction.

Since $N_{1}=G_{p}\left\{A_{(\mathbf{0 1})}^{p^{\left(\theta_{1}-1\right)}}\right\}$ is still non-trivial and normal in $H$, we form $H / N_{1}$ and hence

$$
\operatorname{Var}\left(H / N_{1}\right)=\left[\xi_{1}, \xi_{2}, \cdots, \xi_{(s 1-1)}, \xi_{(s 1)}, \cdots, \xi_{(s 2)}, \cdots, \xi_{(s n)}\right]
$$

where $\xi_{(s 1)} \leqq e_{1}-1, \xi_{(82)} \leqq e_{2}-1, \cdots, \xi_{(s n)} \leqq e_{n}-1$. Now since $H$ belongs to the variety formed by its proper quotient groups, and $\operatorname{Var}(H)=\mathfrak{B}$, there must then be at least one other proper normal subgroup $\mathrm{N}_{2}$ of $\mathrm{H}$ such 
that $\operatorname{Var}\left(H / N_{2}\right)=\left[\eta_{1}, \eta_{2}, \cdots, \eta_{c}\right]$ where $\eta_{(s 1)}=e_{1}$. But since $\eta_{1} \geqq \eta_{2} \geqq \cdots \eta_{c}$, this forces $\eta_{1}=\eta_{2}=\cdots=\eta_{(s)}=e_{1}$. But now if any $\eta_{(s i)}<e_{i}$; then

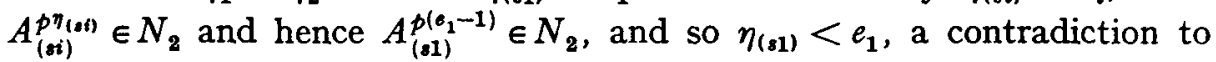
$\eta_{(a 1)}=e_{1}$. Thus $\eta_{(s i)}=e_{i}$ for each $i$, and this forces $\operatorname{Var}\left(H / N_{2}\right)=\mathfrak{B}$, a contradiction to the choice of $H$. Thus $H$ is critical, and the theorem is proved.

\section{Appendix}

For the purpose of proving Theorem 4, we needed the particular group $G$, the recipe for which is given herewith.

Take the $c$ cyclic groups $C_{i}=G p\left\{A_{i} \mid A_{i}^{p^{\alpha_{i}}}=1\right\} i=1,2, \cdots, c$, and form their direct product $P$. We now split-extend this direct product by the cyclic group $B=G p\left\{b \mid b^{\alpha_{1}}=1\right\}$ by using the mapping $A_{1}^{b}=A_{1} A_{2}, \cdots, A_{c-1}^{b}=A_{c-1} A_{c}, A_{c}^{b}=A_{c}$ which defines an automorphism of the direct product. That this has the order $p^{\alpha}$ follows from $A_{i}^{b^{k}}=A_{i} A_{i+1}^{k} A_{i+2}^{\left(\begin{array}{c}k \\ 2\end{array}\right)} \cdots A_{c}^{\left(\begin{array}{c}k \\ c-i\end{array}\right)}$ (which follows from the commuting of the $A_{i}$ in $P$ ) and the facts $p>c$ and $\alpha_{2} \geqq \alpha_{3} \geqq \cdots \geqq \alpha_{c}$. The mapping itself gives immediately $\left(A_{i}, b\right)=A_{i+1}$ for $i<c$, and the structure of $P$ gives $\left(A_{i}, A_{j}\right)=1$ for all $(i, j)$. The resulting group is then isomorphic to $G$ by identifying $A_{1}$ with $a$. By adopting the obvious normal form $b^{t} \prod_{i=1}^{c} A_{i}^{r_{i}}$ for an element of $G$, from which any element of $\gamma_{k}(G)$ is of the form $\prod_{i=k}^{c} A_{i}^{t_{i}}, k>1$, it is clear that $G$ is metabelian, of class $c$, satisfies the basis laws of $\left[\alpha_{1}, \alpha_{2}, \cdots, \alpha_{c}\right]$, and that these indices are minimal for the laws of $G$.

\section{Acknowledgements}

Grateful acknowledgements are due to J. A. Lambert for calling my attention to an explicit formula for the Stirling numbers, to P. M. Neumann for pointing out flaws in an original version of Lemma 2 , and to the referee for pointing out flaws in an original version of Theorem 4. Grateful acknowledgement is especially due to Ian D. Macdonald for continuing encouragement and help throughout.

\section{References}

[1] Various Sources; e.g. M. Abramowitz and I. A. Stegun, 'Handbook of Mathematical Functions' (National Bureau of Standards, 1964).

[2] G. Baumslag, B. H. Neumann, Hanna Neumann and Peter M. Neumann, 'On varieties generated by a finitely generated group', Math. $Z$. 86 (1964), 93-122.

[3] W. Brisley, 'A Problem of D. W. Barnes', Proc. Internat. Conf. Theory of Groups, A.N.U., Canberra, 1965. (Gordon and Breach, New York, 1966.) 
[4] N. D. Gupta and M. F. Newman, 'On metabelian groups', J. Aust. Math. Soc., 6 (1966), 362-368.

[5] Graham Higman, 'Some remarks on varieties of groups', Quarterly J. Math. Oxford (2) 10 (1959), $165-178$.

[6] L. G. Kovács and M. F. Newman, 'Minimal Verbal Subgroups', Proc. Cambridge Philos. Soc.

[7] I. D. Macdonald, 'A theorem about critical p-groups', Proc. Internat. Cont. Theory of Groups, A.N.U., Canberra, 1965, (Gordon and Breach, New York, 1966).

[8] Hanna Neumann, Varieties of Groups (Duplicated lecture notes, Manchester College of Science and Technology, 1963).

[9] Hanna Neumann (Book to be published by Springer).

[10] P. M. Weichsel, 'On critical p-groups', Proc. Lond. Math. Soc., 3rd series, 14 (1964), 83-100.

Department of Mathematics

University of Newcastle 